\title{
Three-dimensional reconstruction facilitates off-clamp laparoscopic partial nephrectomy for stage cT1b renal tumors
}

\author{
Xiaorong Wu ${ }^{1 \#}$, Chao Shen ${ }^{1 \#}$, Guangyu Wu ${ }^{2}$, Chen Jiang ${ }^{1}$, Qibo Fu ${ }^{1}$, Dongming Liu ${ }^{1}$, Wei Xue ${ }^{1}$ \\ ${ }^{1}$ Department of Urology, ${ }^{2}$ Department of Radiology, Ren Ji Hospital, School of Medicine, Shanghai Jiao Tong University, Shanghai 200127, China \\ Contributions: (I) Conception and design: X Wu, C Shen, D Liu, W Xue; (II) Administrative support: DL, W Xue; (III) Provision of study materials \\ or patients: X Wu, C Shen, G Wu, C Jiang, Q Fu, D Liu, W Xue; (IV) Collection and assembly of data: X Wu, C Shen, G Wu, C Jiang, Q Fu; (V) \\ Data analysis and interpretation: X Wu, C Shen; (VI) Manuscript writing: All authors; (VII) Final approval of manuscript: All authors. \\ \#These authors contributed equally to this work. \\ Correspondence to: Dongming Liu; Wei Xue. Department of Urology, Ren Ji Hospital, School of Medicine, Shanghai Jiao Tong University, 160 Pu \\ Jian Road, Shanghai 200127, China. Email: rjliudongming@sina.com; xuewei@renji.com.
}

\begin{abstract}
Background: This study aimed to evaluate the role of three-dimensional (3D) reconstruction of T1b renal tumors in the off-clamp laparoscopic partial nephrectomy (LPN).

Methods: A total of 40 consecutive patients undergoing LPN for stage cT1b renal tumor between January 2018 and July 2018 were included. Twenty received off-clamp LPN under the guidance of 3D reconstruction (3D group), and remaining 20 underwent off-clamp LPN under the guidance of conventional computer tomography arteriography (CTA group). The demographics, perioperative characteristics and renal function were compared between groups.
\end{abstract}

Results: All the procedures were performed successfully without conversion to main renal artery clamping. There were no significant differences in the age, gender, body mass index (BMI), tumor size, and RENAL score between two groups. The mean operation time (OT) was significantly shorter and estimated blood loss markedly less in the 3D group than in the CTA group. Incidence of postoperative complications was $5 \%$ in the $3 \mathrm{D}$ group and $10 \%$ in the CTA group $(\mathrm{P}>0.05)$. 3D reconstruction of renal tumors resulted in more accurate dissection of the tumor artery (90.9\%) as compared to conventional CTA $(81.5 \%)$. All the patients had negative surgical margins. There was no significant difference in the estimated glomerular filtration rate (eGFR) before and after surgery between two groups.

Conclusions: 3D reconstruction is beneficial for the resection of cT1b renal tumor and tumor-specific feeding arteries in the off-clamp LPN.

Keywords: Laparoscopy; partial nephrectomy; renal neoplasm; three-dimensional reconstruction; zero ischemia

Submitted Nov 17, 2019. Accepted for publication Dec 30, 2019.

doi: $10.21037 /$ tcr.2020.01.49

View this article at: http://dx.doi.org/10.21037/tcr.2020.01.49

\section{Introduction}

Nephron sparing surgery (NSS) with the advantage of preserving renal function has become the "gold standard" in the treatment of cT1a and selective cT1b renal tumors (1). Laparoscopic partial nephrectomy (LPN) has been widely accepted since its first introduction (2). However, the management of cT1b renal tumors with LPN is still a technical challenge due to its complexity.
With the development of technology, some approaches have been used in the treatment of cT1b renal tumors, including laparoscopic, robotic assisted NSS and ablation techniques (such as cryoablation and microwave ablation) (3-5). Minimizing or even eliminating warm ischemia (WI) injury and improving the functional outcomes after NSS are the major concerns of the urologists. Several technique modifications have also been developed to reduce or minimize WI during partial nephrectomy $(\mathrm{PN})$, including 
the segmental renal artery clamping, zero ischemia minimally invasive $\mathrm{PN}$ and near-infrared fluorescence imaging with indocyanine green (ICG) in robot-assisted partial nephrectomy (6), which are also suitable for the treatment of selective cT1b renal tumors $(7,8)$.

As the concept of "precision surgery" raised in the management of genitourinary cancers, a detailed and casespecific understanding of surgical anatomy is of great importance to surgical planning and even orientation (9). Antonelli et al. evaluated the differences in the perception of renal anatomy between holographic reconstruction versus computed tomography in patients who were candidate to $\mathrm{PN}$, and results revealed that holographic reconstruction was useful for the preoperative surgical planning before PN to ease the understanding of anatomy (10). Recently, advances in the $3 \mathrm{D}$ reconstruction and printing technology have fueled a rapidly growing interest in its applications in the field of urology (11). Currently, the experience on the use of $3 \mathrm{D}$ reconstruction to facilitate zero-ischemia $\mathrm{PN}$, especially for cT1b renal tumors, remains sparse, and the role of $3 \mathrm{D}$ reconstruction is still needed to be defined.

Herein, we reported our preliminary experience on the use of $3 \mathrm{D}$ reconstruction of $\mathrm{cT} 1 \mathrm{~b}$ renal tumors in the off-clamp LPN as compared to conventional computer tomography arteriography (CTA).

\section{Methods}

Forty consecutive patients who underwent retroperitoneal off-clamp LPN in our hospital between January 2018 and July 2018 were retrospectively analyzed. The patients were diagnosed with stage cT1bN0M0 sporadic renal tumor. Patients undergoing LPN for multiple renal tumors, bilateral renal tumors, or tumors of solitary kidney were excluded from this study. The study was approved by the Institutional Review Board of Ren Ji Hospital (No. 2017-091) and informed consent was taken from all the patients. Before surgery, all the patients received contrast-enhanced CT CTA, and additional 3D reconstruction was conducted in 20 patients.

CTA was performed using a 64-multidetector computed tomography scanner (VCT Light Speed, GE Healthcare, Pittsburgh, USA). Patients were administered with $1,000 \mathrm{~mL}$ of water before the CTA. Four phase images were obtained in a craniocaudal direction. The scanning covered the area from the diaphragm to the lower kidney poles (non-enhanced, arterial and portal phases) and from the diaphragm to the symphysis pubis (delayed nephrographic excretory phase). Contrast-enhanced images were obtained after intravenous administration of $150 \mathrm{~mL}$ of non-ionic contrast medium (Iopamiro, Bracco, Milan, Italy). The current was $110-380 \mathrm{~mA}$, the slice thickness was $1.25 \mathrm{~mm}$, and the pitch was 1.375 . The images in the non-enhanced nephrographic and excretory phase were reconstructed as $1.25-\mathrm{mm}$ sections. The images in the arterial phase were reconstructed at a $0.725-\mathrm{mm}$ interval.

Images in DICOM format were processed with a dedicated software by Mimics, 3-matic and Magics (Materialise, Belgium). Then, a 3D virtual model was established on the basis of CTA examination. The reconstruction focused on the semitransparent kidney, the tumor characteristics and the arterial vasculature, as well as the collecting system. The segmentation of kidney surface was performed in the arterial phase images with the automated threshold segmentation method using Hounsfield units. The renal pedicle and tumor feeding arteries were reconstructed in the arterial phase by using the dynamic region growing method. Then, the virtual renovascular-tumor models were reviewed by both urologists and bioengineers to evaluate the accuracy of models in comparison to the CTA images (Figure 1). The $3 \mathrm{D}$ model and the relative interactive $3 \mathrm{D}$ images were then created.

All operations were performed by the same laparoscopic surgeon with extensive LPN experience (D Liu). Patients received general anesthesia and lied in a lateral decubitus position. All the procedures were performed using a retroperitoneal approach. Four ports were made in the lumbar region. In the CTA group, off-clamp LPN was performed under the guidance of CTA images. In the $3 \mathrm{D}$ group, snapshots of $3 \mathrm{D}$ images from various angle views (e.g., lateral, anterior and posterior) were created and presented to the surgeon preoperatively and intraoperatively. After the tumor was completely exposed, the parenchyma was then incised, and the tumor was excised along its capsule with a margin of 1 - to $2-\mathrm{mm}$ normal parenchyma by blunt dissection, the dissection was maintained at the relatively avascular intrarenal plane. On reaching the target vessels of the tumor, operation was done with Hem-o-lok clips (Figure 2). Hemostasis was achieved with point-specific suturing, and the incised calyces were repaired. Then, the defected parenchyma was closed via continuous suturing with Hem-o-lok clips.

Patients were followed up via hospital visit, reviewing medical records or telephoning. The general information and tumor characteristics including RENAL score were 

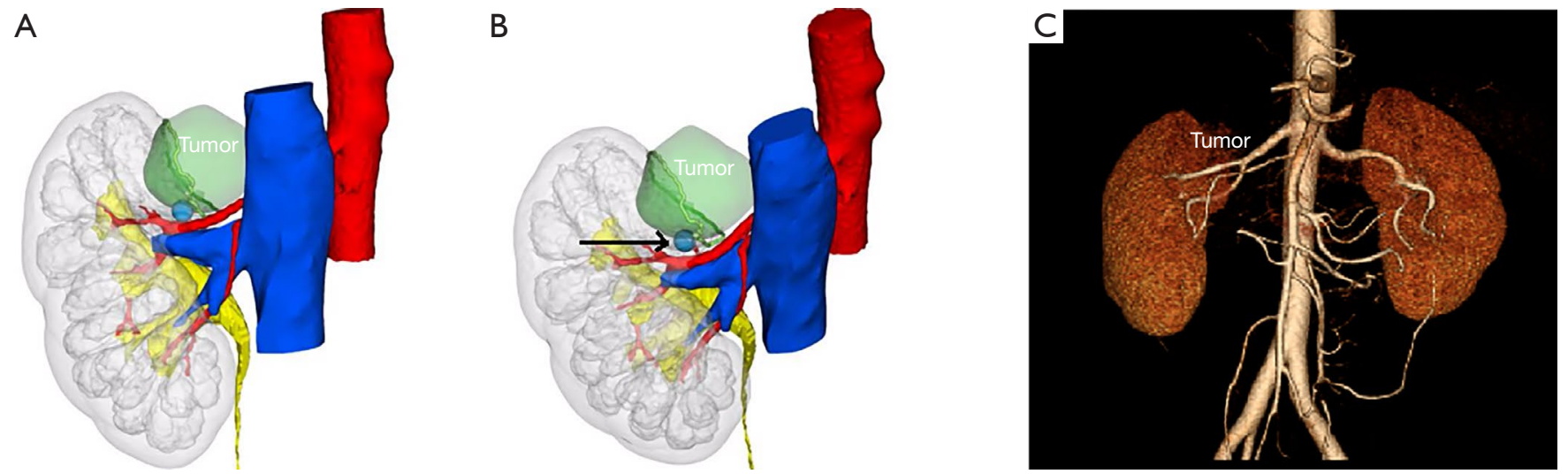

Figure 1 Virtual renovascular-tumor models and conventional CTA images. (A) Three-dimensional (3D) reconstruction images of renovascular tumor with five key surgical anatomies, including 3D surface-rendered renal tumor (green), semitransparent kidney (grey), and $3 \mathrm{D}$ course of the extra- and intrarenal arteries (red), renal veins (blue) and collecting system (yellow); (B) the reconstructed images were rotated to display the number and location of tumor feeding arteries. There was one feeding artery in this tumor (black arrow); (C) conventional CTA could identify extrarenal arteries and the tumor, but failed to determine the intrarenal arteries. CTA, computed tomography angiography.
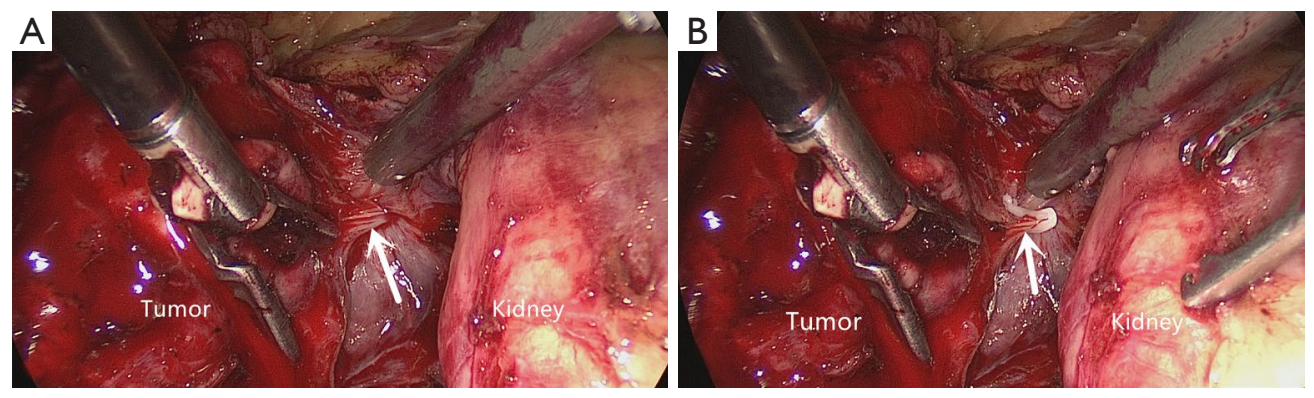

Figure 2 The details of tumor enucleation during off-clamp laparoscopic partial nephrectomy. (A) The tumor was excised by blunt dissection along its capsule with a margin of 1- to 2-mm normal parenchyma to maintain the dissection at the relatively avascular intrarenal plane. Under the guidance of 3D reconstructed images, the tumor specific feeding artery (white arrow) could be identified in the surgery; (B) once the tumor feeding artery was identified, it was clamped with the Hem-o-lok clips (white arrow).

collected (12). All complications within 30 days after surgery were considered being related to the surgery, and staged according to the 2004 Clavien-Dindo grading system (13). The estimated glomerular filtration rate (eGFR) was calculated preoperatively and 12 months postoperatively according to the Modification of Diet in Renal Disease Equation (14). All patients in this study were required for follow-up for at least 3 months. Follow-up was conducted at 3 and 6 months after surgery (since they were discharged) and once every 6 months thereafter. Routine blood test and chest and abdomen enhanced CT were performed during follow up. Recurrence was defined as any new enhancement $(10 \mathrm{HU})$ at 3 months after surgery. Statistical analysis was done with SPSS version 21.0. The continuous variables with normal distribution were compared with Student $t$-test, while the Wilcoxon rank sum was used for the non-normal distributed continuous variables. The comparisons of categorized demographic or clinical variables among groups were done with Pearson Chi-square or Fisher exact test. Two-sided $\mathrm{P}<0.05$ was considered statistically significant.

\section{Results}

A total of 40 patients were included in this study. The baseline characteristics of included patients are shown in Table 1 . The mean age was $58.95 \pm 11.69$ years in the $3 \mathrm{D}$ 
Table 1 Patients' preoperative demographics

\begin{tabular}{|c|c|c|c|}
\hline Variables & $3 D$ group $(n=20)$ & CTA group $(n=20)$ & $P$ value \\
\hline Male & 15 [75] & $14[70]$ & \\
\hline Female & $5[25]$ & $6[30]$ & \\
\hline Age (years, mean $\pm S D$ ) & $58.95 \pm 11.69$ & $54.15 \pm 11.9$ & 0.206 \\
\hline Tumor size $(\mathrm{cm}$, mean $\pm \mathrm{SD})$ & $5.05 \pm 0.63$ & $4.95 \pm 0.67$ & 0.628 \\
\hline Side of the kidney, $\mathrm{n}$ [\%] & & & 0.327 \\
\hline Left & $11[55]$ & $14[70]$ & \\
\hline Right & $9[45]$ & $6[30]$ & \\
\hline Middle & $11[55]$ & $12[60]$ & \\
\hline Lower pole & $5[25]$ & $3[15]$ & \\
\hline RENAL score & & & 0.964 \\
\hline $4-6$ & 3 & 3 & \\
\hline $7-9$ & 14 & 15 & \\
\hline $10-12$ & 3 & 2 & \\
\hline
\end{tabular}

SD, standard deviation; RENAL Score: (R)adius, (E)xophytic/endophytic properties of the tumor, (N)earness of the deepest portion of the tumor to the collecting system or sinus, (A)nterior (a)/posterior ( $p$ ) descriptor and the (L)ocation relative to the polar line. BMI, body mass index; CTA, computed tomography angiography.

group and $54.15 \pm 11.9$ years in the CTA group. The mean tumor size was 5.05 and $4.95 \mathrm{~cm}$ in the $3 \mathrm{D}$ group and CTA group, respectively, showing no significant difference $(\mathrm{P}>0.05)$. There were also no significant differences between two groups in the gender, body mass index (BMI), tumor location, and RENAL score.

The number of tumor feeding arteries was identified preoperatively and confirmed intraoperatively (Table 2). Based on the preoperative $3 \mathrm{D}$ reconstructed images, 9 patients had only one tumor feeding artery, 9 had two and others 2 had three. The conventional CTA revealed that 13 patients had one tumor feeding artery and 7 had two, showing no significant difference between two groups. However, under the guidance of 3D reconstructed images, more tumor feeding arteries were accurately identified during the surgery as compared to that under the guidance of conventional CTA $(\mathrm{P}=0.043)$. Despite there was a difference in tumor feeding artery orientation between $3 \mathrm{D}$ group and CTA group (90.9\% vs. $81.5 \%)$, it was not statistically significant $(\mathrm{P}=0.285)$.

Off-clamp LPN was successfully performed in all the patients without conversion to main renal artery clamping, open surgery and radical nephrectomy. The mean operation time (OT) was $127.56 \pm 18.67$ and $140.02 \pm 11.06 \mathrm{~min}$ in the $3 \mathrm{D}$ group and CTA group, respectively $(\mathrm{P}=0.014)$. The average estimated blood loss (EBL) was less and the hospital stay was significantly shorter in the $3 \mathrm{D}$ group as compared to the CTA group ( $\mathrm{P}=0.005$ and 0.033 , respectively). No statistical differences were found between two groups in the major surgical complications, mean serum eGFRs before surgery and at 1 year after surgery, and change in mean eGFR (Table 3).

Histopathological examination showed clear-cell renal cell carcinoma (RCC) (3D group: $n=17$; CTA group: $n=16$ ), chromophobe RCC (3D group: $n=1$; CTA group: $n=3$ ), and papillary RCC (3D group: $n=3$; CTA group: $n=2$ ). There were no significant differences in the subtypes and Fuhrman grade between two groups $(\mathrm{P}>0.05)$. The surgical margins 
Table 2 Tumor feeding arteries according to 3D reconstruction of renovascular-tumor, conventional CTA and surgically confirmed findings in 40 patients

\begin{tabular}{lcc}
\hline Variables & 3D group & CTA group \\
\hline Preoperative findings of tumor feeding arteries, $\mathrm{n}[\%]$ & & 0.226 \\
1 & $9[45]$ & $9[65]$ \\
2 & $2[10]$ & $7[35]$ \\
3 & & $0(0)$ \\
No. surgically confirmed findings, $\mathrm{n}[\%]$ & $11[55]$ & $18[90]$ \\
1 & $8[40]$ & $2[10]$ \\
2 & $1[5]$ & 0.043 \\
3 & $90.9 \%(30 / 33)$ & $81.5 \%(22 / 27)$ \\
\hline
\end{tabular}

3D, three-dimension; CTA, computed tomography angiography.

Table 3 Patients' postoperative characteristics

\begin{tabular}{|c|c|c|c|}
\hline Variables & $3 D$ group $(n=20)$ & CTA group $(n=20)$ & $P$ \\
\hline Estimated blood loss $(\mathrm{mL}$, mean $\pm \mathrm{SD})$ & $125.52 \pm 55.30$ & $184.53 \pm 69.17$ & 0.005 \\
\hline Hospital stay (days, mean \pm SD) & $5.61 \pm 1.15$ & $6.40 \pm 1.27$ & 0.033 \\
\hline Pathologic subtype, n (\%) & & & 0.506 \\
\hline Chromophobe & $1[5]$ & $3[15]$ & \\
\hline Papillary & $2[10]$ & $1[5]$ & \\
\hline Fuhrman grade, n (\%) & & & 0.913 \\
\hline I & $4[20]$ & $4[20]$ & \\
\hline Complications, n (\%) & & & 0.387 \\
\hline Fever & $1[5]$ & 0 & \\
\hline Hematuria & 0 & $2[10]$ & \\
\hline eGFR pre-operation $\left(\mathrm{mL} / \mathrm{min} / 1.73 \mathrm{~m}^{2}\right.$, mean $\left.\pm \mathrm{SD}\right)$ & $106.49 \pm 14.63$ & $112.50 \pm 15.97$ & 0.222 \\
\hline eGFR 1 year post-operation $\left(\mathrm{mL} / \mathrm{min} / 1.73 \mathrm{~m}^{2}\right.$, mean $\left.\pm \mathrm{SD}\right)$ & $96.70 \pm 12.41$ & $103.06 \pm 13.76$ & 0.133 \\
\hline eGFR change $\left(\mathrm{mL} / \mathrm{min} / 1.73 \mathrm{~m}^{2}\right.$, mean $\left.\pm \mathrm{SD}\right)$ & $-9.79 \pm 9.25$ & $-10.44 \pm 10.67$ & 0.837 \\
\hline
\end{tabular}

3D, three-dimension; CTA, computed tomography angiography; eGFR, estimated glomerular filtration rate; SD, standard deviation. 
were negative in all cases, and no renal capsule invasion was found. The median duration of follow-up was 15 months in two groups, and local recurrence or metastasis was not found.

\section{Discussion}

Mir et al. introduced the concept of "global renal damage" in $\mathrm{PN}$, in which the factors determining the quantity as well as the quality of preserved parenchyma included patients' re-operative features and surgical factors, such as baseline kidney function, ischemia time and type and resection and reconstruction technique $(15,16)$. The major concerns of LPN are the complete removal of renal tumor and the effective hemostasis with short WI time (15). Several techniques have been developed to achieve better renal function, such as segmental artery clamping, super selective embolization, and radiofrequency and microwave ablation $(7,17)$. In recent years, zero ischemic LPN has introduced to eliminate WI injury and has been popularized among the urologic communities (18-20).

Gill et al. (19) for the first time introduced the concept of zero ischemia LPN. They tried to eliminate global renal ischemia by meticulous microdissection of tertiary or quaternary renal arterial branches feeding the tumor. However, dissecting a tertiary or quaternary arterial branch from the renal hilum is time-consuming and technically difficult. The uncertainty of the relationship between the tumor and the feeding arteries makes off-clamp LPN even more difficult, especially for the T1b renal tumors (19). Therefore, preoperative understanding of the anatomy of each tumor is of great importance.

CTA has been the preferred imaging modality for the preoperative evaluation of renal tumors. Advances in the CT and computer technology allow the generation of high quality images of the renal vasculature, parenchyma, tumor and collecting system at any plane (21). It can also accurately identify the number of extrarenal arteries. However, CTA has a poor capacity to display the precise anatomical interrelationship and the precise location of various intrarenal tributaries. In addition, the kidney, tumor and renal vessels on the conventional CTA are typically unclear, which makes it impossible to visualize the intrarenal interrelationships between the tumor and its feeding arteries (22). The understanding of relevant intrarenal anatomy during LPN currently is wholly based on the preoperative CT images combined with intraoperative laparoscopic visualization and two-dimensional ultrasonography (22).
Thus, it is imperative to develop new techniques for radiologic guidance (23).

In this study, the patients in the $3 \mathrm{D}$ group underwent $3 \mathrm{D}$ reconstruction of the renal tumor. The $3 \mathrm{D}$ images included $3 \mathrm{D}$ surface rendered semitransparent renal tumor, transparent kidney, and 3D course of extraand intrarenal arteries, and the collecting system. The number and location of tumor feeding arteries were determined much more easily, and during the surgery, the $3 \mathrm{D}$ reconstructed images of renovascular tumor resulted in more accurate dissection of target tumor artery than in the CTA group. For cT1b renal tumors, especially the endophytic renal tumors challenging laparoscopy, $3 \mathrm{D}$ reconstructed images are helpful for the precise identification of the collecting system and tumor specific feeding arteries, thus facilitating the off-clamp LPN and reducing the OT and EBL.

Porpiglia $e t$ al. assessed the face and content validity of 3D virtual models of prostates and kidneys used before robotassisted prostate cancer and nephron-sparing surgery $(24,25)$. Their results revealed that $3 \mathrm{D}$ printing technology was a useful tool for the surgical planning and physician education/ training, as well as patient counseling. Based on our experience, $3 \mathrm{D}$ reconstruction and printing models could help surgeons during their learning curve in laparoscopic NSS. It has been shown that the application of $3 \mathrm{D}$ virtual models in robot-assisted radical prostatectomy (RARP) results in a reduction of the positive surgical margin rate (26). Thus, 3D reconstruction techniques have several advantages as compared to conventional CTA. It can clearly display the intrarenal arterial tree and the detailed anatomical interrelationships among tumor, collecting system and intrarenal arteries. The reconstructed images can also be rotated to clarify the number and location of tumor feeding arteries (Figure 1A,B). In addition, 3D reconstruction images can be used for preoperative evaluation by surgeons, which provides reference for the pre-operative determination of appropriate dissection strategy and also for the intraoperative real-time orientation, therefore avoiding invisible injury to other interlobar arteries during off-clamp LPN. Moreover, it's also useful for the physician education/training and patient counseling (24).

There were also several limitations in this study. The $3 \mathrm{D}$ reconstruction technique based on CT images is not applicable to magnetic resonance imaging. Thus, patients who can't receive enhanced CT are excluded from the study on 3D reconstruction. As shown in Table 2, the tumor feeding arteries were confirmed more accurately under 
the guidance of $3 \mathrm{D}$ reconstruction technique, but the rate of accurately tumor feeding arteries orientation was only $90.9 \%$, which was similar to that with the guidance based on conventional CTA. In addition, the mean diameter was $5 \mathrm{~cm}$ in the present study, and whether this strategy is also applicable in the management of larger T1b renal tumors is still unclear. In the management of T1b or even complicated renal tumors with off-clamp LPN, robot assisted surgery may be helpful for better tumor resection and renorrhaphy $(25,27-29)$, but experience is lacking in this field. 3D reconstructions are gaining a wide attention in NSS planning and orientation, however, they were usually studied on common 2D flat supports, with limitations regarding real depth comprehension and interaction. Checcucci et al. proposed 3D mixed reality holograms as a useful and interesting tool for the preoperative setting before $\mathrm{PN}$, in the direction of an ever more precise surgery (30). These new technologies should be recommended in our future study. This was also a retrospective single-center study with small sample size, and patients received only short-term follow up. Thus, we could not confirm the risk of systematic recurrence free survival rate. In addition, the tumor contact with the excretory system was not assessed in our study. Thus, more randomized, controlled trials are needed to confirm our findings in more patients. Of note, the expertise of the surgeon and the costs to obtain a $3 \mathrm{D}$ rendering may limit the application of our technique in routine clinical practice.

In conclusion, our study indicates that $3 \mathrm{D}$ reconstruction technique is a useful tool and can provide comprehensive information about the intrarenal interrelationships between the $\mathrm{cT} 1 \mathrm{~b}$ renal tumor and its feeding arteries. The model seems to facilitate tumor feeding artery orientation and dissection, therefore improving the surgical outcome after off-clamp LPN.

\section{Acknowledgments}

Funding: This study was supported by the Three-yearaction-project of Shanghai Hospital Development Center (No: 16CR3062B), the Medical-Engineering cross fund of Shanghai Jiao Tong University (No: YG2017QN46) and the Doctorial Innovation Fund of School of Medicine, Shanghai Jiao Tong University (No: CBXJ201804).

\section{Footnote}

Conflicts of Interest: All authors have completed the ICMJE uniform disclosure form (available at http://dx.doi. org/10.21037/tcr.2020.01.49). The authors have no conflicts of interest to declare.

Ethical Statement: The authors are accountable for all aspects of the work in ensuring that questions related to the accuracy or integrity of any part of the work are appropriately investigated and resolved. The study was conducted in accordance with the Declaration of Helsinki (as revised in 2013). The study was approved by the Institutional Review Board of Ren Ji Hospital (No. 2017091) and informed consent was taken from all the patients.

Open Access Statement: This is an Open Access article distributed in accordance with the Creative Commons Attribution-NonCommercial-NoDerivs 4.0 International License (CC BY-NC-ND 4.0), which permits the noncommercial replication and distribution of the article with the strict proviso that no changes or edits are made and the original work is properly cited (including links to both the formal publication through the relevant DOI and the license). See: https://creativecommons.org/licenses/by-nc-nd/4.0/.

\section{References}

1. Ljungberg B, Albiges L, Abu-Ghanem Y, et al. European Association of Urology Guidelines on Renal Cell Carcinoma: The 2019 Update. Eur Urol 2019;75:799-810.

2. Ng AM, Shah PH, Kavoussi LR. Laparoscopic Partial Nephrectomy: A Narrative Review and Comparison with Open and Robotic Partial Nephrectomy. J Endourol 2017;31:976-84.

3. Heinze A, Larcher A, Umari P, et al. Assessing perioperative, functional and oncological outcomes of patients with imperative versus elective indications for robot-assisted partial nephrectomy: Results from a highvolume center. Int J Urol 2018;25:826-31.

4. Deng W, Chen L, Wang Y, et al. Cryoablation versus Partial Nephrectomy for Clinical Stage T1 Renal Masses: A Systematic Review and Meta-Analysis. J Cancer 2019;10:1226-36.

5. Wells SA, Wheeler KM, Mithqal A, et al. Percutaneous microwave ablation of $\mathrm{T} 1 \mathrm{a}$ and $\mathrm{T} 1 \mathrm{~b}$ renal cell carcinoma: short-term efficacy and complications with emphasis on tumor complexity and single session treatment. Abdom Radiol (NY) 2016;41:1203-11.

6. Veccia A, Antonelli A, Hampton LJ, et al. Near-infrared Fluorescence Imaging with Indocyanine Green in Robotassisted Partial Nephrectomy: Pooled Analysis of Comparative 
Studies. Eur Urol Focus 2019. [Epub ahead of print].

7. Shao P, Tang L, Li P, et al. Application of a vasculature model and standardization of the renal hilar approach in laparoscopic partial nephrectomy for precise segmental artery clamping. Eur Urol 2013;63:1072-81.

8. Anderson BG, Potretzke AM, Du K, et al. Comparing Offclamp and On-clamp Robot-assisted Partial Nephrectomy: A Prospective Randomized Trial. Urology 2019;126:102-9.

9. Autorino R, Porpiglia F, Dasgupta P, et al. Precision surgery and genitourinary cancers. Eur J Surg Oncol 2017;43:893-908.

10. Antonelli A, Veccia A, Palumbo C, et al. Holographic Reconstructions for Preoperative Planning before Partial Nephrectomy: A Head-to-Head Comparison with Standard CT Scan. Urol Int 2019;102:212-7.

11. Youssef RF, Spradling K, Yoon R, et al. Applications of three-dimensional printing technology in urological practice. BJU Int 2015;116:697-702.

12. Kutikov A, Uzzo RG. The R.E.N.A.L. nephrometry score: a comprehensive standardized system for quantitating renal tumor size, location and depth. J Urol 2009;182:844-53.

13. Dindo D, Demartines N, Clavien PA. Classification of surgical complications: a new proposal with evaluation in a cohort of 6336 patients and results of a survey. Ann Surg 2004;240:205-13.

14. Levey AS, Stevens LA, Schmid CH, et al. A new equation to estimate glomerular filtration rate. Ann Intern Med 2009; 150:604-12.

15. Blair BM, Lehman EB, Jafri SM, et al. Predicted versus observed 30-day perioperative outcomes using the ACS NSQIP surgical risk calculator in patients undergoing partial nephrectomy for renal cell carcinoma. Int Urol Nephrol 2018;50:1249-56.

16. Mir MC, Autorino R, Porpiglia F. Ischemia time and beyond: the concept of global renal damage. Minerva Urol Nefrol 2018;70:447-9.

17. Huang J, Zhang J, Wang Y, et al. Comparing Zero Ischemia Laparoscopic Radio Frequency Ablation Assisted Tumor Enucleation and Laparoscopic Partial Nephrectomy for Clinical T1a Renal Tumor: A Randomized Clinical Trial. J Urol 2016;195:1677-83.

18. Wang HK, Qin XJ, Ma CG, et al. Nephrometry scoreguided off-clamp laparoscopic partial nephrectomy: patient selection and short-time functional results. World J Surg Oncol 2016;14:163.

19. Gill IS, Eisenberg MS, Aron M, et al. "Zero ischemia" partial nephrectomy: novel laparoscopic and robotic technique. Eur Urol 2011;59:128-34.
20. Abdel Raheem A, Santok GD, Kim LHC, et al. Off-Clamp Robot-Assisted Partial Nephrectomy: How Far Shall We Proceed? J Laparoendosc Adv Surg Tech A 2018;28:579-85.

21. Shao P, Tang L, Li P, et al. Precise segmental renal artery clamping under the guidance of dual-source computed tomography angiography during laparoscopic partial nephrectomy. Eur Urol 2012;62:1001-8.

22. Ukimura O, Nakamoto M, Gill IS. Three-dimensional reconstruction of renovascular-tumor anatomy to facilitate zero-ischemia partial nephrectomy. Eur Urol 2012;61:211-7.

23. Ukimura O, Gill IS. Imaging-assisted endoscopic surgery: Cleveland Clinic experience. J Endourol 2008;22:803-10.

24. Porpiglia F, Bertolo R, Checcucci E, et al. Development and validation of $3 \mathrm{D}$ printed virtual models for robotassisted radical prostatectomy and partial nephrectomy: urologists' and patients' perception. World J Urol 2018;36:201-7.

25. Malkoc E, Ramirez D, Kara O, et al. Robotic and open partial nephrectomy for localized renal tumors larger than 7 cm: a single-center experience. World J Urol 2017;35:781-7.

26. Porpiglia F, Manfredi M, Checcucci E, et al. 3D prostate MRI reconstruction for congitive robot assisted radical prostatectomy: Is it able to reduce the positive surgical margin rate? European Urology Supplements 2017;16:e110-e111.

27. Antonelli A, Veccia A, Francavilla S, et al. On-clamp versus off-clamp robotic partial nephrectomy: A systematic review and meta-analysis. Urologia 2019;86:52-62.

28. Antonelli A, Cindolo L, Sandri M, et al. Predictors of the Transition from Off to On Clamp Approach during Ongoing Robotic Partial Nephrectomy: Data from the CLOCK Randomized Clinical Trial. J Urol 2019;202:62-8.

29. Bertolo R, Simone G, Garisto J, et al. Off-clamp vs on-clamp robotic partial nephrectomy: Perioperative, functional and oncological outcomes from a propensityscore matching between two high-volume centers. Eur J Surg Oncol 2019;45:1232-7.

30. Checcucci E, Amparore D, Pecoraro A, et al. 3D mixed reality holograms for preoperative surgical planning of nephron-sparing surgery: evaluation of surgeons' perception. Minerva Urol Nefrol 2019. [Epub ahead of print].

Cite this article as: $\mathrm{Wu} \mathrm{X}$, Shen $\mathrm{C}, \mathrm{Wu}$ G, Jiang C, Fu Q, Liu D, Xue W. Three-dimensional reconstruction facilitates off-clamp laparoscopic partial nephrectomy for stage cT1b renal tumors. Transl Cancer Res 2020;9(3):1528-1535. doi: 10.21037/tcr.2020.01.49 